\title{
Infrared Spectroscopy of Molecules in Disks
}

\author{
Joan Najita \\ National Optical Astronomy Observatory, Tucson, AZ 85719, USA \\ email:najita@noao.edu
}

\begin{abstract}
Disks surrounding young stars play a fundamental role in the formation of stars and planets. Accretion through disks is believed to be responsible for the build up of stellar masses, and the gas and dust in disks is a reservoir for the potential formation of planets. As a result, one of the motivations for observing the inner regions of disks (i.e., the region within $10 \mathrm{AU}$ ) is to obtain clues to the processes that govern how stars and planets form. Significant progress has been made over the last decade in probing the inner regions of gaseous disks through the use of infrared molecular transitions. I discuss the observational tools that are currently available to study the gaseous component. These tools can be used to explore the evolution of gas in the inner disk and thereby help us to understand the processes of giant and terrestrial planet formation. These same tools may also be used to place constraints on the physical mechanisms that drive the disk accretion process.
\end{abstract}

Keywords. stars: pre-main-sequence - accretion disks — planetary systems: protoplanetary disks — planetary systems: formation

\section{Introduction}

Circumstellar disks are believed to form as a natural consequence of the collapse of molecular cloud cores. Due to the finite angular momentum of cloud cores, all of the infalling matter cannot fall directly onto the star; instead a large fraction must accrete through a disk before reaching the star. A long-standing problem regarding this phase of star formation is the nature of the physical mechanism that mediates the redistribution of angular momentum, thereby allowing accretion to occur. Several accretion mechanisms have been proposed, perhaps the foremost of which is the magnetorotational instability (Balbus \& Hawley 1991; Stone et al. 2000), although other possibilities exist (e.g., the global baroclinic instability-Klahr \& Bodenheimer 2003). Despite the significant theoretical progress that has been made in identifying plausible accretion mechanisms, observational evidence that any of these processes are actually active in disks has been difficult to come by. In this contribution, I suggest how new opportunities to study the inner region of disks may allow us to probe the nature of the accretion process.

Similarly, observations of the inner disk region may provide insight into the physical processes that govern planet formation and the resulting planetary architectures (i.e., planetary masses, orbital radii, and eccentricities). These clues are likely to be valuable given that the planet formation process appears to be more complex than was once believed. In the 10 years since their discovery, the extrasolar planets have come to span an ever increasing range in orbital properties, demonstrating a remarkable diversity that has, in turn, inspired multiple theories of their origins. The possibility that multiple theories may account for the orbital properties of the extrasolar planets drives us to search beyond planetary architectures for clues to their origins, clues that may be contained in the formation environments of planets, i.e., disks surrounding young stars.

For example, we might attempt to measure the gas dissipation timescale in the inner disk in order to constrain pathways for the formation of giant planets. Measurements of 
the gas dissipation timescale would also constrain the efficiency of orbital migration and eccentricity evolution for both terrestrial and giant planets. In this contribution, I discuss some of the molecular probes currently available to probe the inner disk as well as current thermal-chemical modeling of the atmospheres of inner disks. As a specific application, I illustrate how these tools could be used to measure the gas dissipation timescale in inner disks and thereby place constraints on the outcome of terrestrial planet formation.

\section{Molecular Probes of Gaseous Inner Disks}

We might first wonder what situations might give rise to emission lines from the inner disk, since disks are typically expected to be very optically thick in the continuum due to the high column density of gas and dust. (For example, the total column density of the minimum mass solar nebula at $1 \mathrm{AU}$ is $\sim 1500 \mathrm{~g} \mathrm{~cm}^{-2}$.) We can nevertheless expect to detect emission lines in several important cases. At early times, irradiation by the central star, or other heating processes, may heat the surface of the disk, creating a warm "disk chromosphere" that will produce emission lines. Emission lines are also expected at late times, once the inner disk has evolved to either low total column density (e.g., due to disk dissipation) or low continuum optical depth (e.g., due to grain growth or the formation of rocky bodies).

Given that line emission can plausibly arise from inner disks, we might ask what spectral features are to be expected? The temperatures involved range from several $1000 \mathrm{~K}$ at the inner disk edge to $\sim 100 \mathrm{~K}$ in the giant planet region of the disk $(5-10 \mathrm{AU})$. In addition, the disks are massive so they should be characterized by high densities. These conditions suggest that molecules should be good probes of disks since they should be abundant in the gas phase at these temperatures, and at high density they should be excited enough to produce a ro-vibrational spectrum in the infrared.

This expectation is borne out in that there are now multiple molecular probes of the inner disk region. These include the $\mathrm{CO}$ overtone lines, which probe the region from the inner disk edge at $\sim 0.05 \mathrm{AU}$ out to a few $0.1 \mathrm{AU}$ (e.g., Najita et al. 2000 for a review) and the CO fundamental lines, which probe the region from the inner disk edge out to larger distances of a few AU, i.e., the terrestrial planet region of the disk (e.g., Najita et al. 2003). Tracers that probe somewhat larger radii than the CO overtone lines include water ro-vibrational lines in the $K$-band (e.g., Carr et al. 2004) and $\mathrm{OH}$ fundamental vibrational lines in the $L$-band (Carr et al., in preparation). In addition, transitions of molecular hydrogen at UV, NIR, and MIR wavelengths (e.g., Herczeg et al. 2002; Bary et al. 2003; Richter et al. 2002) are together expected to probe both the terrestrial and giant planet regions of the disk.

Among these, CO overtone emission (e.g., Carr 1989, Scoville et al. 1979) is perhaps the diagnostic first recognized to probe the inner disk region. High-resolution spectra of $v=2-0$ bandhead emission from young stars at $2.3 \mu \mathrm{m}$ typically shows the characteristic shape expected for bandhead emission from a rotating disk (e.g., Carr et al. 1993; Chandler et al. 1993; Najita et al. 1996). Such bandhead profiles are seen in young stars ranging in mass from $\sim 1 \mathrm{M}_{\odot}$ to $\sim 10 \mathrm{M}_{\odot}$ (e.g., Najita et al. 2000; Blum et al. 2004). These results provide good evidence for rotating inner disks around young stars of low to high mass.

If we look in more detail at the shape of the underlying rotationally broadened line, we find that the emission is broad (typically $>100 \mathrm{~km} \mathrm{~s}^{-1}$ ). This suggests that the inner radius for the emission arises very close to the star, at $\sim 0.05 \mathrm{AU}$. The double-peaked profiles indicate that the emission arises over a limited range of radii, out to $\sim 0.3 \mathrm{AU}$ 
in low-mass stars. These small radii are consistent with the high deduced excitation temperature of the emission over this range of radii $(\sim 1500-5000 \mathrm{~K})$.

An interesting aspect of the overtone bandhead is that it is made up of closely spaced lines with varying inter-line spacing and optical depth. This makes it possible to distinguish the intrinsic line broadening of the emitting gas from macroscopic motions such as rotation. Using spectral synthesis modeling, we are therefore able to deduce that the lines are suprathermally broadened, with line widths approximately Mach 2 (Carr et al. 2004; Najita et al. 1996). This suggests that disk atmospheres are turbulent. This may be a consequence of turbulent angular momentum transport in disks, as is expected in the case of both the magnetorotational instability and the global baroclinic instability.

Another interesting molecular probe of the inner disk is the ro-vibrational transitions of water. In the $K$-band, $\mathrm{CO}$ overtone emission is often accompanied by individual emission lines of water blueward of the bandhead (Carr et al. 2004; Najita et al. 2000; Thi \& Bik 2005). Velocity resolved spectra show that the width of the CO emission is always broader than the width of the water emission. In addition, spectral synthesis modeling shows that the excitation temperature of the $\mathrm{CO}$ emission is larger than that of the water emission. These properties are consistent with a common origin for the water and CO emission in a differentially rotating disk with an outwardly decreasing temperature profile. This is because the lower dissociation temperature of water $(\sim 2500 \mathrm{~K}$ in inner disks $)$ allows the water emission to extend in only to a modest radius ( $>0.05 \mathrm{AU})$. In contrast, the much higher dissociation temperature of $\mathrm{CO}(\sim 5000 \mathrm{~K}$ in inner disks $)$ allows the $\mathrm{CO}$ emission to extend in to much smaller radii $(\sim 0.05 \mathrm{AU})$, to higher velocities and higher temperatures.

Recent spectral synthesis modeling that fits both the $\mathrm{CO}$ and water emission finds that the relative abundance of water to $\mathrm{CO}$ is low, a factor of $2-10$ below the chemical equilibrium value in low-mass stars (Carr et al. 2004; see Thi \& Bik 2005 in the context

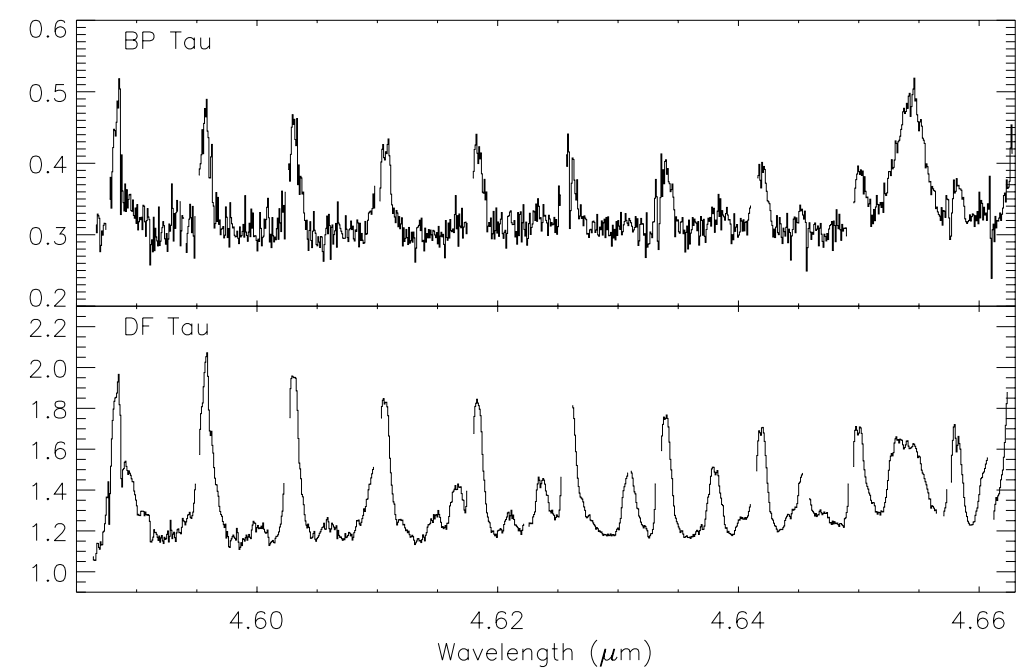

Figure 1. CO fundamental emission from accreting young stars. Regions of strong telluric absorption have been excised from the plot. The centrally peaked line profiles show that the fundamental transitions probe a wide range of disk radii, from inner disk edge at $\sim 0.05 \mathrm{AU}$ out to 1-2 AU, i.e., the terrestrial planet region of the disk. The emission spectrum can be rich (e.g., DF Tau), displaying lines from a range of vibrational levels (e.g., $v=1-0,2-1,3-2$ ) as well as lines of ${ }^{13} \mathrm{CO}$. 
of high-mass stars). The low ratio may reflect strong vertical abundance gradients that result from the external irradiation of the disk (see $\S 3$ ).

$\mathrm{CO}$ fundamental emission at $4.6 \mu \mathrm{m}$ is interesting as a disk diagnostic because it is common: it is detected in almost all accreting young stars (e.g., Najita et al. 2003). This is perhaps a consequence of the much larger A-values for the fundamental lines compared to the $\mathrm{CO}$ overtone lines. In contrast to the overtone lines which are double-peaked, the line profiles for the fundamental emission are broad and centrally peaked. This suggests that the $\mathrm{CO}$ fundamental transitions probe a wide range of radii, from the inner disk edge (at $\sim 0.05 \mathrm{AU}$ ) out to $1-2 \mathrm{AU}$, i.e., the terrestrial planet region of the disk.

The emission spectra typically show emission lines from a range of vibrational states; ${ }^{13} \mathrm{CO}$ can also be detected when the emission is bright (Fig. 1). The ability to study a wide range of vibrational states as well as isotopic species within a limited spectral range suggests the utility of $\mathrm{CO}$ fundamental emission in probing a wide range of gas temperatures and column densities. Indeed, an excitation analysis of the emission provides evidence for surprisingly warm gas $(\gtrsim 1000 \mathrm{~K})$ in the terrestrial planet region of disks surrounding low-mass stars. These temperatures are much higher than the dust temperatures expected for the same region of the disk (e.g., D'Alessio et al. 1998).

\section{Models of Gaseous Atmospheres of Inner Disks}

These warm temperatures can be understood in the context of current thermal-chemical models for the inner disk region. For example, in the models of Glassgold, Igea \& Najita (2004), we find that both the gas and dust in the disk atmosphere experience temperature inversions, but with the gas achieving much higher surface temperatures than the dust. In these models, the dust is heated by stellar (primarily optical) photons, whereas the gas is heating by stellar X-rays at the disk surface and by accretion-related heating processes at intermediate heights. Possible accretion-related processes include in situ accretional heating and the dissipation of turbulence induced by a stellar wind blowing over the disk surface. These processes produce a temperature inversion in the gaseous atmosphere over a column density of $\sim 10^{22} \mathrm{~cm}^{-2}$. At larger column densities (and higher densities), the gas temperature is driven down to the dust temperature due to efficient gas-grain coupling (Fig. 2).

If we look at the chemistry that takes place within this vertical temperature structure, we find that a small abundance of $\mathrm{H}_{2}$ forms high in the atmosphere through the $\mathrm{H}^{-}$and 3 -body pathways. This allows the formation of CO at a column density of $\sim 10^{21} \mathrm{~cm}^{-2}$. In contrast, the full conversion from atomic $\mathrm{H}$ to molecular $\mathrm{H}_{2}$ is made further down in the atmosphere, at a column density of $\sim 6 \times 10^{21} \mathrm{~cm}^{-2}$, with more complex molecules such as water forming deeper in the disk, primarily through neutral reactions. This produces a disk atmosphere with layered molecular abundances. Similar descriptions of warm disk atmospheres with layered molecular abundances have been made for the outer disk region (e.g., Kamp \& Dullemond 2004; Jonkheid et al. 2004; see Kamp, this volume).

Importantly, the region of the disk atmosphere over which the gas and dust temperatures differ includes the region currently accessible to observations (e.g., using the CO transitions). Thus, using models of disk atmospheres that consider the potential thermal decoupling of gas and dust can be important in interpreting observations. Indeed, looking at the temperature at which $\mathrm{CO}$ forms, the temperature at $1 \mathrm{AU}$ is $\sim 1000 \mathrm{~K}$, which is similar to the observations. Perhaps interestingly, the CO is abundant in a region in which hydrogen is predominantly in atomic form. This is favorable for exciting the CO transitions since the collisional cross-section for exciting $\mathrm{CO}$ with atomic hydrogen is larger than the cross-section for exciting $\mathrm{CO}$ with $\mathrm{H}_{2}$. 

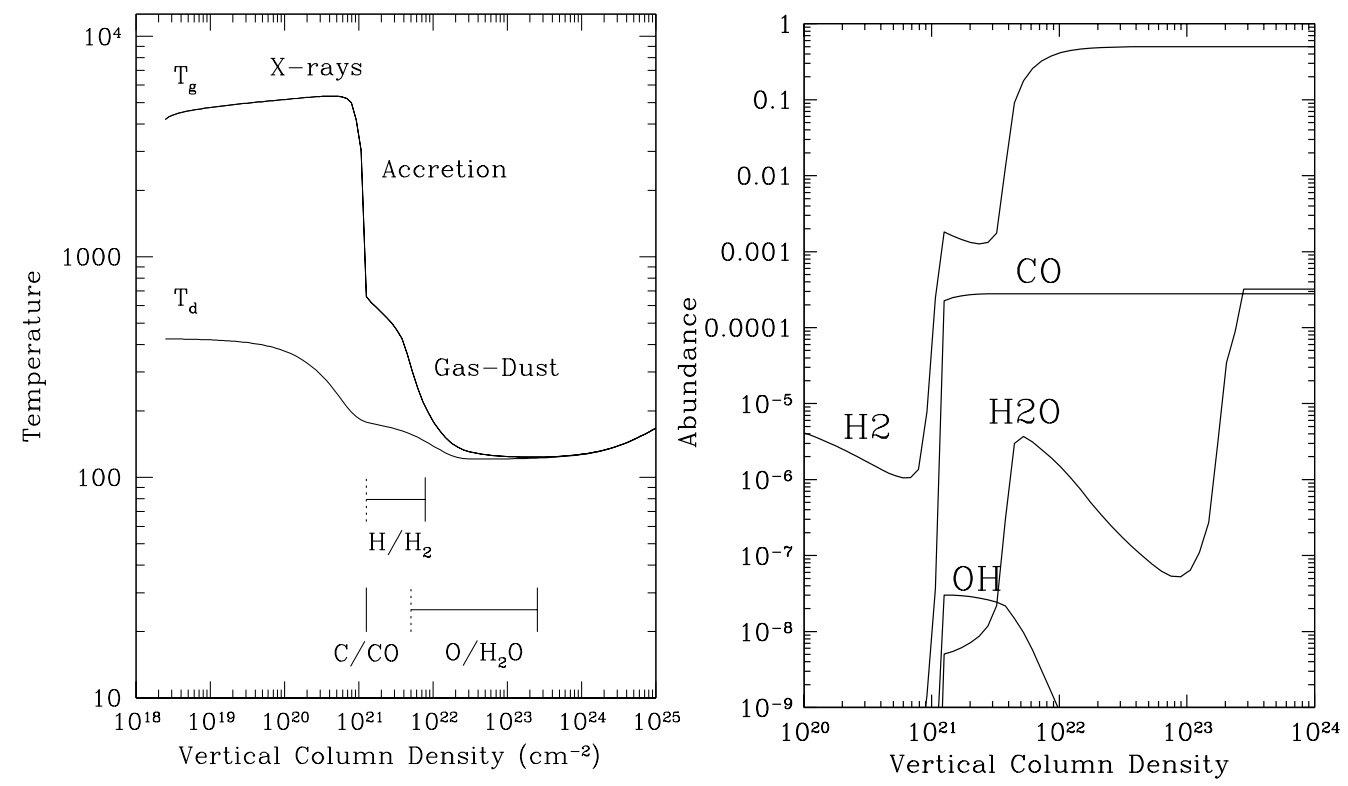

Figure 2. Left: Vertical temperature structure in the atmosphere of a disk surrounding an accreting young star at a radial distance of 1 AU (Glassgold et al. 2004). The gas and dust components both experience vertical temperature inversions, with the gas achieving much higher temperatures than the dust. Right: In the accompanying vertical chemical structure, a modest abundance of $\mathrm{H}_{2}\left(x\left(\mathrm{H}_{2}\right)=10^{-3}\right)$ is achieved at a vertical column density of $\sim 10^{21} \mathrm{~cm}^{-2}$, which allows $\mathrm{CO}$ to form at a similar column density. Full conversion to $\mathrm{H}_{2}$ is achieved at a much larger column density $\sim 10^{22} \mathrm{~cm}^{-2}$. Similarly, a modest water abundance $\left(x\left(\mathrm{H}_{2} \mathrm{O}\right)=3 \times 10^{-6}\right)$ is achieved at $\sim 5 \times 10^{21} \mathrm{~cm}^{-2}$, with an asymptotic abundance $\left(x\left(\mathrm{H}_{2} \mathrm{O}\right)=3 \times 10^{-4}\right)$ achieved at much higher column densities $\left(>10^{23} \mathrm{~cm}^{-2}\right)$.

There is additional evidence for warm molecular gas in the inner disk that comes from from UV observations of fluorescent molecular hydrogen emission. As shown by Herczeg et al. (2002) in the case of the young star TW Hya, Ly $\alpha$ is observed to pump $\mathrm{H}_{2}$ from excited energy levels $\sim 2000 \mathrm{~K}$ above ground, showing that the excited gas is warm, with a characteristic $\mathrm{H}_{2}$ column density of $\sim 10^{18} \mathrm{~cm}^{-2}$. It is interesting to think about the implications of this column density in the context of the thermal-chemical models discussed in the previous section.

In the Glassgold et al. models, we predict that the $\mathrm{H}_{2}$ abundance at the disk surface is small $\sim 10^{-3}$ (Fig. 2). This implies that the measured $\mathrm{H}_{2}$ column density of $\sim 10^{18} \mathrm{~cm}^{-2}$ for TW Hya corresponds to a total hydrogen column density of $\sim 10^{21} \mathrm{~cm}^{-2}$. Since this is the column density over which $\operatorname{Ly} \alpha$ is found to excite the gas, the dust in the disk atmosphere must be depleted relative to the gas in order for Ly $\alpha$ to penetrate this far. Thus, observations of this kind, when combined with gaseous disk atmosphere models, can provide one of the few ways of constraining the dust-to-gas ratio in disk atmospheres. The low dust-to-gas ratio may be taken as evidence for significant grain growth or settling in the disk atmosphere of TW Hya. This is in agreement with the low dust column density in the inner disk that is inferred from the spectral energy distribution for this source (Calvet et al. 2002). The gas observations show that the gas may be less depleted than the small dust grains in the inner region of the disk.

It is also interesting to look at the relative abundances of water and $\mathrm{CO}$ in these models. At $1 \mathrm{AU}$, we find that water is at least 100 times less abundant than $\mathrm{CO}$ in the 
disk atmosphere (Fig. 2). This is much less than the ratio inferred from spectroscopy at smaller disk radii, where water is $\sim 3$ times less abundant than CO (see $\S 2$ ). It would therefore be interesting to model the disk atmosphere at smaller radii to see if larger water abundances are predicted there. If not, perhaps the discrepancy between the observations and the thermal-chemical predictions for static disk atmospheres is evidence for strong vertical mixing which dredges up abundant water from deeper in the disk to the disk surface.

\section{Gas in the Terrestrial Planet Region of Disks}

Finally, I wanted to describe how we might use gas-phase diagnostics of the inner disk region to probe the lifetime of gas in the terrestrial planet region. This issue is of interest because residual gas in the disk can affect the outcome of terrestrial planet formation, i.e., the resulting masses and eccentricities of planets and their consequent habitability. For example, in the picture of terrestrial planet formation described by Kominami \& Ida (2002), only gas column densities in a narrow range around $\sim 1 \mathrm{~g} \mathrm{~cm}^{-2}$ are likely to produce planets with Earth-like masses and eccentricities. With gas column densities much larger than this, protoplanets (the building blocks of terrestrial planets) experience significant gravitational gas drag and are not able to acquire the eccentricities that are needed for them to collide and form more massive objects. Conversely, if the gas column density is much lower than $1 \mathrm{~g} \mathrm{~cm}^{-2}$, protoplanets can easily acquire the eccentricities that are needed for them to collide, producing a massive eccentric object. But then the gravitational gas drag is insufficient to re-circularize the orbit. As a result, there is only a narrow range of gas column densities around $1 \mathrm{~g} \mathrm{~cm}^{-2}$ that can produce planets with Earth-like masses (i.e., massive enough to hold on to life-sustaining atmospheres) and the low eccentricities that we associate with habitability on Earth.

To understand how often this situation occurs during the period of terrestrial planet formation, John Carr, Bob Mathieu, and I have been carrying out a survey for CO

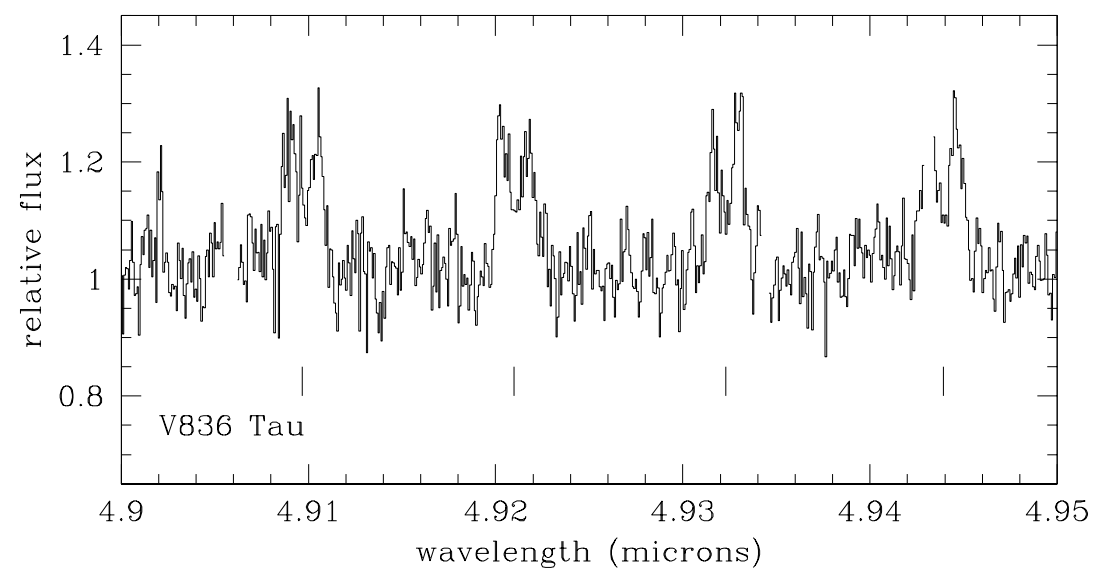

Figure 3. CO fundamental emission from the transitional T Tauri star V836 Tau, a system that is developing an optically thin inner disk. The spectra show $v=1-0$ emission from high- $J$ P-branch lines at $4.9 \mu \mathrm{m}$. Regions of strong telluric absorption have been excised from the plot. The vertical lines mark the approximate CO line centers at the velocity of the star. The velocity widths of the lines indicate that the emitting gas is located within a few AU of the star, and the relative strengths of the lines suggest optically thick emission. Thus a large reservoir of gas may be present in the inner disk despite the weak infrared excess from this portion of the disk. 


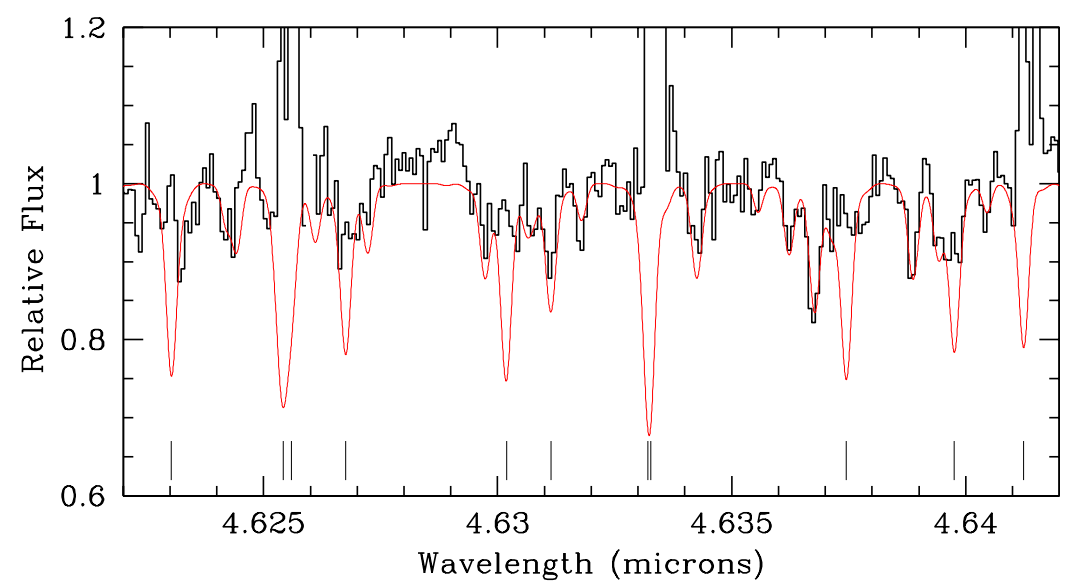

Figure 4. Spectrum of the transitional T Tauri star TW Hya in the $4.6 \mu \mathrm{m}$ region (histogram), focusing on the structure in the continuum. The strong emission lines that extend above the plotted region are $v=1-0$ low- $J$ R-branch CO fundamental lines. For comparison, the predicted stellar spectrum for TW Hya is also shown (gray line). The model stellar spectrum fits many of the weaker features seen in the continuum. However, it predicts stronger stellar $\mathrm{CO}$ absorption in the low vibrational transitions (indicated by the lower vertical lines) than is seen in the measured spectrum. This suggests that the stellar photospheric spectrum is veiled by $\mathrm{CO}$ emission from warm disk gas.

fundamental emisison from young stars (see Najita 2004). CO is attractive for this purpose because it probes small column densities of gas $\left(10^{-4}-1 \mathrm{~g} \mathrm{~cm}^{-2}\right)$ covering the range of interest. We are targeting young stars with optically thin inner disks, because we want to know how much gas is left once disks become optically thin as a result of the formation of planetesimals, protoplanets, or larger bodies. Thus, our sample is made up of weaklined $\mathrm{T}$ Tauri stars (stars with no evidence for disks or stellar accretion) and transitional $\mathrm{T}$ Tauri stars (stars with evidence for outer dust disks and a range of accretion rates).

Obtaining an accurate answer to our problem is challenging for several reasons. Firstly, we of course need reliable thermal-chemical models in order to convert any observed emission or upper limit into a total gas column density. Secondly, the strength of CO emission is known to correlate with the accretion rate in sources with optically thick inner disks (Najita et al. 2003). This suggests that the lower accretion rates that are known to characterize our sample may result in less heating of the upper disk atmosphere (see $\S 3$ and Fig. 2) and therefore intrinsically weaker $\mathrm{CO}$ emission. Care may therefore be needed to detect such weak emission.

Nevertheless, thus far we have a few obvious detections of fairly strong emission. This shows that gas may survive in inner disks even after the continuum becomes optically thin. The detections include the T Tauri star V836 Tau (Fig. 3), a $\sim 3$ Myr old system (Siess et al. 1999), with an $\mathrm{H} \alpha$ equivalent width of $9 \AA$ (Herbig \& Bell 1988), and an estimated accretion rate of $\sim 4 \times 10^{-10} \mathrm{M}_{\odot} \mathrm{yr}^{-1}$ (Hartigan et al. 1995; Gullbring et al. 1998). CO emission is also detected from TW Hya (Fig. 4; see also Rettig et al. 2004, and G. Blake personal communication), an $\sim 8$ Myr old T Tauri star with a mass accretion rate of $4 \times 10^{-10}-2 \times 10^{-9} \mathrm{M}_{\odot} \mathrm{yr}^{-1}$ (Muzerolle et al. 2000; Alencar \& Basri 2000).

Detecting weaker emission from or placing stringent limits on the gas content in the remainder of the sample will require attention to issues such as correcting for absorption in stellar photospheres. For example, in the case of TW Hya (Fig. 4), comparison of the observed spectrum with a model stellar atmosphere appropriate for its spectral type 
indicates that $\mathrm{CO}$ absorption in the stellar atmosphere is "veiled" by $\mathrm{CO}$ emission from the disk. Correcting for CO absorption in the stellar photosphere would uncover emission in higher vibrational transitions of CO. The warmer gas temperatures implied by the detection of emission from higher vibrational levels is consistent with the warm molecular gas temperature that is inferred for this system from UV fluorescent $\mathrm{H}_{2}$ emission (Herczeg et al. 2002).

Since we have yet to reach a conclusion about the lifetime of gas in the terrestrial planet region of disks based on spectroscopic studies, I wanted to try to guess at an answer using other data. A useful data set for this purpose is stellar accretion rates, which have been measured for many young stars (e.g., Gullbring et al. 1998; Hartmann et al. 1998; Muzerolle et al. 1998, 2000; Sicilia-Aguilar et al. 2005). If we assume that the accretion onto the star is fed by steady accretion in the disk, then the disk column density $\Sigma \propto \dot{\mathrm{M}}_{*} / \alpha \mathrm{T}$ where $\dot{\mathrm{M}}_{*}$ is the accretion rate onto the star, $T$ is the disk temperature, and the viscosity is parameterized as $\nu=\alpha c_{s} H$ where $c_{s}$ is the sound speed and $H$ is the disk scale height. Using a relation of this form, we can infer the disk column density given the stellar accretion rate and a value for the viscosity parameter $\alpha$.

Many of the sources in our sample have stellar accretion rates of a few $\times 10^{-10} \mathrm{M}_{\odot} \mathrm{yr}^{-1}$ (e.g., V836 Tau) or upper limits on the accretion rate that are in this range. For $\alpha=$ 0.01 , a value that is commonly adopted in the literature, accretion rates in this range correspond to column densities at $1 \mathrm{AU}$ of a few $\mathrm{g} \mathrm{cm}^{-2}$, i.e., a dynamically significant column density. Since sources with this level of accretion are detected at ages up to $10 \mathrm{Myr}$ or older, it seems plausible that dynamically significant reservoirs of gas can survive for long enough to influence the outcome of terrestrial planet formation.

Actually measuring total disk gas column densities using the approach described above can confirm this speculation. Once a measurement is in hand, we would know both the accretion rate and the disk column density. This would imply a value of $\alpha$, which would be another way of constraining the physical mechanism that drives disk accretion.

\section{Summary and Future Directions}

In summary, infrared spectroscopy of the gas in disks has provided several clues to the processes that govern star and planet formation. It provides evidence for differentially rotating inner disks around young stars. It also suggests that the atmospheres of these disks are turbulent. This may indicate that the disk accretion process is itself turbulent.

The measured excitation temperature of molecular tracers in inner disks demonstrates that the gas is typically hotter than the dust in the same region of the disk. In addition, spectroscopy of multiple gas-phase species provides evidence for non-equilibrium molecular abundances in disk atmospheres. These characteristics are in rough agreement with current thermal-chemical models of inner disk atmospheres.

These results suggest several possible future directions. To explore whether the disk accretion process is turbulent, one might try to extend the techniques described in $\S 2$ to other tracers in order to measure the turbulent line width as a function of disk radius and column density. The results could be compared with theoretical predictions for the turbulence in disk atmospheres that is produced as a consequence of, e.g., the MRI instability. Since turbulence at disk surfaces might also be produced as a consequence of a stellar wind blowing over the disk, it would be of interest to see whether these two scenarios can be distinguished on the basis of this kind of observational data.

Another approach to exploring the extent of turbulence in disk atmospheres would be to look in more detail at the chemical abundances in disks. As described in $\S 3$, if relative abundances cannot be explained by static atmosphere models, this might suggest 
that vertical mixing (produced by turbulence) is playing a role. Yet another approach is to measure total gas column densities in dissipating disks that show signs of ongoing accretion. As described in $\S 4$, knowing both the mass accretion rate and the disk column density can provide a constraint on the viscosity parameter $\alpha$.

On the topic of the gas dissipation timescale in disks, there is evidence that gaseous reservoirs in the terrestrial planet region of disks can survive beyond the timescale for the inner disk to become optically thin in the continuum. Stellar accretion rates suggest that these gaseous reservoirs may be dynamically significant and may survive long enough to influence the outcome of terrestrial planet formation. Understanding whether or not this is in fact true requires improvements in data analysis techniques to optimize for the detection of weak emission as well as improvements in models of disk atmospheres in order to convert detections or upper limits into disk gas column densities. These same concerns are relevant to similar studies, now ongoing, to probe the gas dissipation timescale at larger disk radii, in the giant planet region of disks.

\section{Acknowledgements}

I would like to thank my colleagues John Carr and Al Glassgold, with whom I have discussed many of the issues reviewed in this contribution.

\section{References}

Alencar, S. H. P. \& Basri, G. 2000, Ap. J. 119, 1881

Balbus, S. A. \& Hawley, J. F. 1991, Ap. J. 376, 214

Bary, J. S., Weintraub, D. A., \& Kastner, J. H. 2003, Ap. J. 586, 1138

Beckwith, S. V. W., Sargent, A. I., Chini, R. S., \& Guesten, R. 1990, A. J. 99, 924

Bergin, E. et al. 2004, Ap. J. 614, L133

Blake, G. A. \& Boogert, A. C. A. 2004, Ap. J. 606, L73

Blum, R. D., Barbosa, C. L., Damineli, A., Conti, P. S., \& Ridgway, S. 2004, Ap. J. 617, 1167

Bodenheimer, P. \& Lin, D. N. C. 2002, Annual Review of Earth and Planetary Sciences 30, 113

Boss, A. P. 1995, Science 276, 1836

Brittain, S. D., Rettig, T. W., Simon, T., Kulesa, C., DiSanti, M. A., \& Dello Russo, N. 2003, Ap. J. 588, 535

Calvet, N., D'Alessio, P., Hartmann, L, Wilner, D., Walsh, A., \& Sitko, M. 2002, Ap. J. 568, 1008

Calvet, N. et al. 2005, A. J. 129, 935

Carr, J. S. 1989, Ap. J. 345, 522

Carr, J. S., Tokunaga, A. T., Najita, J., Shu, F. H., \& Glassgold, A. E. 1993, Ap. J. 411, L37

Carr, J. S., Tokunaga, A. T., \& Najita, J. 2004, Ap. J. 603, 213

Chandler, C. J., Carlstrom, J. E., Scoville, N. Z., Dent, W. R. F., \& Geballe, T. R. 1993, Ap. J. 412, L71

D’Alessio, P., Canto, J., Calvet, N., \& Lizano, S. 1998, Ap. J. 500, 411

Glassgold, A. E., Najita, J., \& Igea, J. 2004, Ap. J. 615, 972

Gorti, U. \& Hollenbach, D. 2004, Ap. J. 613, 424

Gullbring, E., Hartmann, L., Briceño, C., \& Calvet, N. 1998, Ap. J. 492, 323

Hartigan, P., Edwards, S., \& Ghandour, L. 1995, Ap. J. 452, 736

Hartmann, L., Calvet, N., Gullbring, E., \& D'Alessio, P. 1998, Ap. J. 495, 385

Herbig, G. H. \& Bell, K. R. 1988, Lick Obs. Publ. 1111.

Herczeg, G. J., Linsky, J. L., Valenti, J. A., Johns-Krull, C. M., \& Wood, B. E. 2002, Ap. J. 572,310

Jonkheid, B., Faas, F. G. A., van Zadelhoff, G.-J., \& van Dishoeck, E. F. 2004, A\&GA 428, 511

Kamp, I. \& Dullemond, C. P. 2004, Ap. J. 615, 991

Klahr, H. H. \& Bodenheimer, P. 2003, Ap. J. 582, 869

Kominami, J. \& Ida, S. 2002, Icarus 157, 43 
Mayer, L., Quinn, T., Wadsley, J., \& Stadel, J. 2002, Science 298, 1756

Muzerolle, J., Hartmann, L., \& Calvet, N. 1998, A. J. 116, 2965

Muzerolle, J., Calvet, N., Briceño, C., Hartmann, L., \& Hillenbrand, L. 2000, Ap. J. 535, L47

Najita, J., Carr, J. S., Glassgold, A. E., Shu, F. H., \& Tokunaga, A. T. 1996, Ap. J. 462, 919

Najita, J., Edwards, S., Basri, G., \& Carr, J. 2000, in Protostars and Planets IV. ed. V. Mannings, A. P. Boss, \& S. S. Russell (University of Arizona Press), p. 457

Najita, J., Carr, J. S., \& Mathieu, R. D. 2003, Ap. J. 589, 931

Najita, J. 2004, in Star Formation in the Interstellar Medium: In Honor of David Hollenbach, Chris McKee and Frank Shu ASP Conference Proceedings 323, ed. Johnstone, F.C. Adams, D.N.C. Lin, D.A. Neufeld, \& E.C. Ostriker (Astronomical Society of the Pacific), p. 271.

Rettig, T. W., Haywood, J., Simon, T., Brittain, S. D., \& Gibb, E. 2004, Ap. J. 616, L163

Richter, M. J., Jaffe, D. T., Blake, G. A., \& Lacy, J. H. 2002, Ap. J. 572, L161

Sako, S., et al. 2005, Ap. J. 620, 347

Scoville, N. Z., Hall, D. N. B., Ridgway, S. T., \& Kleinmann, S. G. 1979, Ap. J. 232, L121

Sheret, I., Ramsay Howat, S. K., \& Dent, W. R. F. 2003, MNRAS 343, L65

Sicilia-Aguilar, A., Hartmann, L. W., Hernández, J., Briceño, C., \& Calvet, N. 2005, A. J. 130, 188

Siess, L., Forestini, M., \& Bertout, C. 1999, AA 342, 480

Stone, J. M., Gammie, C. F., Balbus, S. A., \& Hawley, J. F. 2000, in Protostars and Planets $I V$.. ed. V. Mannings, A. P. Boss, \& S. S. Russell (University of Arizona Press), p. 589

Thi, W.-F. \& Bik, A. 2005, A\& A 438, 557

\section{Discussion}

WiLson: Have you thought about whether these techniques would be useful for searching for gas in debris disks? Because it would be very interesting to know if those systems have gas an how much gas they have.

NAJITA: That's a good point. I did not have time to mention that indeed several different groups are currently carrying out such searches, not so much with the specific diagnostics that I have been describing as with atomic and molecular transitions in the UV and mid-infrared. As examples of current work in this field, a recent calculation of the expected mid-infrared emission from residual gas in debris disks has been carried out by Gorti \& Hollenbach (2004), and searches for these emission features are underway using Spitzer/IRS (e.g., the "Formation and Evolution of Planetary Systems" Legacy project; M. Meyer, PI) and ground-based telescopes (e.g., Richter et al. 2002; Sheret et al. 2003; Sako et al. 2005).

Forrest: The depletion of dust in the upper layers of T Tauri disks is also indicated by modelling of the infrared $5-35 \mu \mathrm{m}$ spectra by Calvet et al. (2005) and Furlan et al. (2005, in preparation).

NAJITA: Those data certainly provide additional insight. Specifically, the low-resolution spectra tell us about the dust in the disk. Sometimes, as in the case of TW Hya, the spectral energy distribution (SED) indicates a deficit of continuum emission that can be interpreted as an inner hole to the dust opacity. In addition, using a model for how the gas and dust are distributed relative to each other (e.g., with or without settling), one might also be able to use the SED to diagnose the extent of grain depletion relative to the gas. The $\mathrm{UV} \mathrm{H}_{2}$ transitions that I discussed provide a complementary view. They more directly trace the gas component and constrain the gas-to-dust ratio along a specific line of sight. 